\title{
Differences in Clinical and Laboratory Findings Among Graves' Disease, Painless Thyroiditis and Subacute Thyroiditis Patients With Hyperthyroidism
}

\author{
Hidekatsu Yanai $^{\mathrm{a}, \mathrm{b}}$, Mariko Hakoshima ${ }^{\mathrm{a}}$, Hisayuki Katsuyama ${ }^{\mathrm{a}}$
}

\begin{abstract}
Background: Graves' disease, painless thyroiditis and subacute thyroiditis are important in the differential diagnosis for hyperthyroidism in Japan.

Methods: We retrospectively picked up patients who had been diagnosed as having hyperthyroidism, at National Center for Global Health and Medicine Kohnodai Hospital, between January 2010 and October 2018. According to the guideline for diagnosis of Graves' disease, painless thyroiditis and subacute thyroiditis presented by the Japan Thyroid Association, we diagnosed patients as having such diseases. We obtained clinical and laboratory data by using electronic medical records and database after showing the opt-out.
\end{abstract}

Results: Among 91 patients who showed hyperthyroidism, we found 74 patients with Graves' disease including thyroid storm $(n=5)$, and seven with painless thyroiditis and 10 with subacute thyroiditis. Graves' disease patients included a significantly higher percentage of female patients than painless thyroiditis and subacute thyroiditis patients. Serum free triiodothyronine (FT3) level in Graves' disease patients was significantly higher than that in painless thyroiditis and subacute thyroiditis patients. Serum free thyroxine (FT4) level and the ratio of FT3 to FT4 were significantly higher in Graves' disease than in painless thyroiditis. The levels of thyroid autoantibodies were significantly higher in Graves' disease than in other two diseases. Our study showed an increase of white blood cells (WBCs) and percentage of neutrophil in WBC together with a decrease of percentages of lymphocyte and eosinophil in subacute thyroiditis as compared with Graves' disease and painless thyroiditis. Serum albumin level was significantly lower in subacute thyroiditis patients than in Graves' disease patients. Serum C-reactive protein (CRP) level was significantly higher in subacute thyroiditis patients than in Graves' disease patients.

Manuscript submitted April 24, 2019, accepted May 16, 2019

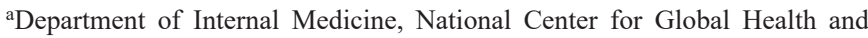
Medicine Kohnodai Hospital, Chiba, Japan

${ }^{\mathrm{b}}$ Corresponding Author: Hidekatsu Yanai, Department of Internal Medicine, National Center for Global Health and Medicine Kohnodai Hospital, 1-7-1 Kohnodai, Ichikawa, Chiba 272-8516, Japan.

Email: dyanai@hospk.ncgm.go.jp

doi: https://doi.org/10.14740/jem572
Conclusions: Present study elucidated characteristic clinical and laboratory findings for patients with Graves' disease, painless thyroiditis and subacute thyroiditis who showed hyperthyroidism.

Keywords: Graves' disease; Hyperthyroidism; Painless thyroiditis; Subacute thyroiditis

\section{Introduction}

Hyperthyroidism is a pathological disorder in which excess thyroid hormone is synthesized and secreted by the thyroid gland, and is characterized by low serum thyroid stimulating hormone (TSH) level and elevated serum levels of free thyroxine (FT4), free triiodothyronine (FT3), or both [1]. The prevalence of overt hyperthyroidism is $0.5-0.8 \%$ in Europe [2], and $0.5 \%$ in the USA [3]. The most common cause of hyperthyroidism in iodine-sufficient areas is Graves' disease, and the cause of Graves' disease is thought to be multifactorial, arising from the loss of immunotolerance and the development of autoantibodies that stimulate thyroid follicular cells by binding to the TSH receptor [1]. Although in iodine-sufficient areas about $80 \%$ of patients with hyperthyroidism have Graves' disease, Plummer's disease (toxic multinodular goiter and hyperfunctioning thyroid nodule) accounts for $50 \%$ of all cases of hyperthyroidism in iodine-deficient areas [4]. In iodine deficient areas, chronic stimulation by TSH causes multinodular autonomous growth and function, leading to hyperthyroidism in middle-aged and elderly subjects. Incidence of Plummer's disease including toxic multinodular goiter in Japan is very low, accounting for $0.3 \%$ of all thyrotoxic patients [5]. Therefore, Graves' disease, painless thyroiditis and subacute thyroiditis are important in the differential diagnosis for hyperthyroidism in Japan.

\section{Materials and Methods}

\section{Study population}

We retrospectively picked up patients who had been diagnosed as having Graves' disease, painless thyroiditis and subacute thyroiditis who showed hyperthyroidism, at National Center 
Table 1. Clinical Differences Among Patients With Graves' Disease, Painless Thyroiditis and Subacute Thyroiditis

\begin{tabular}{|c|c|c|c|c|c|c|}
\hline & & Ives' disease & & less thyroiditis & & ute thyroiditis \\
\hline & $\mathbf{n}$ & Values & $\mathbf{n}$ & Values & n & Values \\
\hline Age (years) & 69 & $49.0 \pm 18.0$ & 7 & $46.9 \pm 21.5$ & 10 & $53.6 \pm 17.3$ \\
\hline Body temperature $\left({ }^{\circ} \mathrm{C}\right)$ & 35 & $36.7 \pm 0.4$ & 7 & $37.0 \pm 0.5$ & 8 & $37.1 \pm 0.8$ \\
\hline Pulse rate (beats/min) & 49 & $102.7 \pm 17.6$ & 6 & $91.5 \pm 15.1$ & 9 & $96.0 \pm 21.6$ \\
\hline
\end{tabular}

Values except for sex indicate mean $\pm S D$. * $P<0.001$ versus patients with Graves' disease and painless thyroiditis.

for Global Health and Medicine Kohnodai Hospital, between January 2010 and October 2018. According to the guideline for diagnosis of Graves' disease, painless thyroiditis and subacute thyroiditis presented by the Japan Thyroid Association, we diagnosed patients as having such diseases. Briefly, we diagnosed patients as having Graves' disease by observing signs of thyrotoxicosis, elevation in serum FT4 and FT3 levels, suppression of TSH and positive for anti-TSH receptor antibody (TRAb). Such a patient shall be probably said to have Graves' disease by this guideline. The guideline for diagnosis of painless thyroiditis includes thyrotoxicosis without pain of the thyroid gland and spontaneous improvement of thyrotoxicosis, elevation of serum FT4, suppression of TSH and negative for TRAb. A patient showing such findings shall be said probably to have painless thyroiditis by the guideline. The guideline for diagnosis of subacute thyroiditis includes a swelling with pain and tenderness in the thyroid gland, elevation of C-reactive protein (CRP), elevation of serum FT4 and suppression of TSH. A patient showing such findings shall be said probably to have subacute thyroiditis. The TRAb had been measured by using the first-, second-, and third-generation TRAb measurement methods between January 2010 and October 2018 in our hospital. Since thyroid storm is a very specific pathological condition, we excluded thyroid storm patients from Graves' disease patients when we compared characteristics among patients with Graves' disease, painless thyroiditis and subacute thyroiditis.

We obtained clinical and laboratory data by using electronic medical records and database after showing the opt-out.

\section{Statistical analysis}

Statistical analyses were performed by using SPSS version 23 (IBM Co., Ltd, Chicago, IL, USA). All values are expressed as the mean \pm standard deviation except for sex. We performed the $t$-test in the comparison among patients with Graves' disease, painless thyroiditis and subacute thyroiditis. P value of $<$ 0.05 was considered statistically significant.

\section{Ethics statement}

Because this study was a retrospective cross-sectional observational study, the opt-out method was adopted. The study protocol was approved by the Ethics Committee of the $\mathrm{Na}$ tional Center for Global Health and Medicine (NCGMG-003120-00), and the study was performed in accordance with the Declaration of Helsinki.

\section{Results}

Among 91 patients who showed hyperthyroidism, we found 74 Graves' disease (81.3\%), seven painless thyroiditis $(7.7 \%)$ and 10 subacute thyroiditis patients $(11.0 \%)$. In patients with Graves' disease, we found five patients complicated with thyroid storm.

Clinical differences among Graves' disease, painless thyroiditis and subacute thyroiditis patients

Clinical differences among Graves' disease, painless thyroiditis and subacute thyroiditis patients were shown in Table 1. There were no significant differences in age, body temperature and pulse rate among such diseases. Graves' disease patients included a significantly higher percentage of female patients than painless thyroiditis and subacute thyroiditis patients.

Differences in TSH, thyroid hormones and thyroid autoantibodies among Graves' disease, painless thyroiditis and subacute thyroiditis patients

Differences in TSH, thyroid hormones and thyroid autoantibodies among Graves' disease, painless thyroiditis and subacute thyroiditis patients were shown in Table 2. There was no significant difference in TSH level among such diseases. Serum FT3 level in Graves' disease patients was significantly higher than that in painless thyroiditis and subacute thyroiditis patients. Serum FT4 level and the ratio of FT3 to FT4 were significantly higher in Graves' disease than in painless thyroiditis. The third-generation TRAb and anti-thyroid peroxidase antibody (TPO-Ab) levels were significantly higher in Graves' disease than in other two diseases. Anti-thyroglobulin antibody (Tg-Ab) level in Graves' disease was significantly higher than that in subacute thyroiditis.

Differences in white blood cell (WBC) fractions among Graves' disease, painless thyroiditis and subacute thyroiditis patients

Differences in WBC fractions among Graves' disease, pain- 
Table 2. Differences in TSH, Thyroid Hormones and Thyroid Autoantibodies Among Patients With Graves' Disease, Painless Thyroiditis and Subacute Thyroiditis

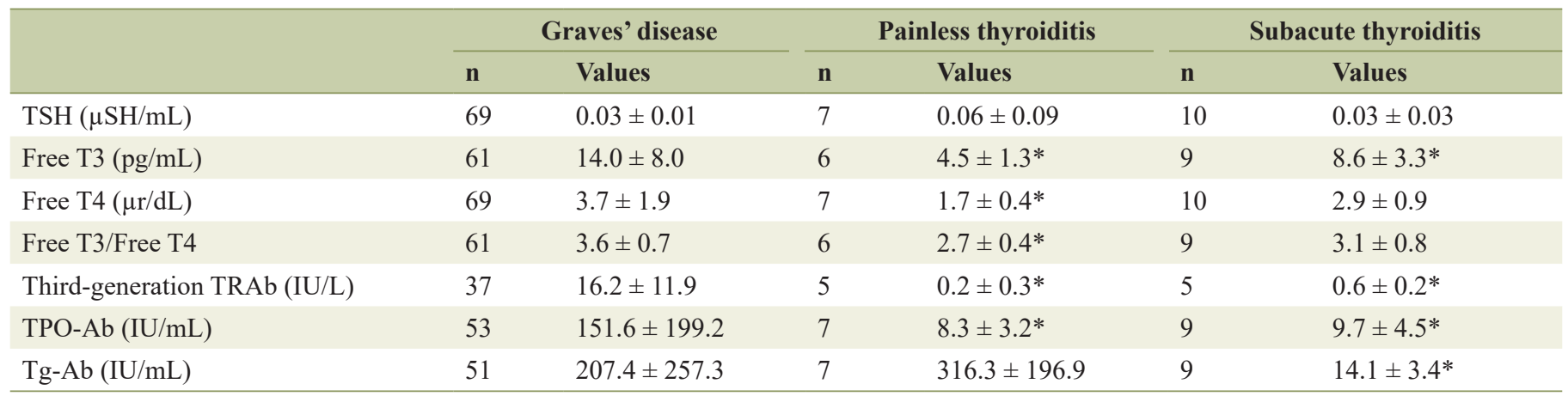

Values indicate mean \pm SD. Tg-Ab: thyroglobulin antibody; TPO-Ab: thyroid peroxidase antibody; TRAb: TSH receptor antibody; TSH: thyroidstimulating hormone; T3: triiodothyronine; T4: thyroxine. ${ }^{*} \mathrm{P} \leq 0.001$ versus patients with Graves' disease.

less thyroiditis and subacute thyroiditis patients were shown in Table 3. WBC count was significantly higher in subacute thyroiditis than in Graves' disease. The percentage of neutrophil among WBC was significantly higher in subacute thyroiditis patients than in Graves' disease patients. Furthermore, the percentages of lymphocyte and eosinophil in subacute thyroiditis were significantly lower than those in Graves' disease.

\section{Biochemical differences among Graves' disease, painless thyroiditis and subacute thyroiditis patients}

Biochemical differences among Graves' disease, painless thyroiditis and subacute thyroiditis patients were shown in Table 4. Serum albumin level was significantly lower in subacute thyroiditis than in Graves' disease. Serum levels of alanine aminotransferase (ALT) and alkaline phosphatase (ALP) were significantly higher in Graves' disease than in painless thyroiditis. Serum creatinine level was significantly lower in Graves' disease than in painless thyroiditis. Serum CRP level was significantly higher in subacute thyroiditis than in Graves' disease.

\section{Discussion}

In Japan, Graves' disease, painless thyroiditis and subacute thyroiditis are important in the differential diagnosis of hy- perthyroidism. Although the measurement of TRAb is very crucial for differential diagnosis for hyperthyroidism and confirmation for diagnosis of Graves' disease, understanding of clinical and laboratory characteristic findings for Graves' disease, painless thyroiditis and subacute thyroiditis patients may lead us to prompt and valid diagnosis.

In present study, the incidence of subacute thyroiditis in female patients was significantly and remarkably lower than that of Graves' disease and painless thyroiditis, and the ratio of women to men was 0.4. In Europe, all subacute thyroiditis patients were female in Austria [6], and the ratio of women to men was 3.2 in Italy [7]. In USA, the ratio of women to men was 3.5 and 6.7 in Minnesota and Hawaii, respectively [8, 9]. In Asia, the ratio of women to men was 14 and 4 in India and Saudi Arabia, respectively $[10,11]$. In Japan, the ratio of women to men was 6, 4 and 7 in 1970s, 1980s and 1996-2004, respectively [12-14]. The incidence of subacute thyroiditis in women varied widely by region and time, which may be explained by the association of development of subacute thyroiditis with various bacterial and viral infections [15-21]. Seasonal changes of the incidence of subacute thyroiditis support our hypothesis [7].

Present study showed that thyroid hormone, especially FT3, was remarkable elevated in Graves' disease. Furthermore, we found that TPO-Ab was increased in Graves' disease, in addition to TRAb. The guideline for diagnosis for Graves' disease presented by the Japan Thyroid Association

Table 3. Differences in White Blood Cell Counts and Fractions Among Patients With Graves' Disease, Painless Thyroiditis and Subacute Thyroiditis

\begin{tabular}{|c|c|c|c|c|c|c|}
\hline & & Iraves' disease & & ainless thyroiditis & & ubacute thyroiditis \\
\hline & $\mathbf{n}$ & Values & n & Values & $\mathbf{n}$ & Values \\
\hline White blood cells $(/ \mu \mathrm{L})$ & 66 & $5,527.3 \pm 1,780.4$ & 7 & $6,385.7 \pm 3,913.0$ & 10 & $9,780.0 \pm 4,013.3^{*}$ \\
\hline Lymphocytes (\%) & 56 & $36.7 \pm 10.8$ & 6 & $27.2 \pm 13.3$ & 7 & $22.8 \pm 11.7^{*}$ \\
\hline Monocytes (\%) & 56 & $7.7 \pm 2.5$ & 6 & $6.3 \pm 2.2$ & 7 & $6.6 \pm 2.1$ \\
\hline
\end{tabular}

Values indicate mean $\pm \mathrm{SD} .{ }^{*} \mathrm{P}<0.05$ versus patients with Graves' disease. 
Table 4. Biochemical Differences Among Patients With Graves' Disease, Painless Thyroiditis and Subacute Thyroiditis

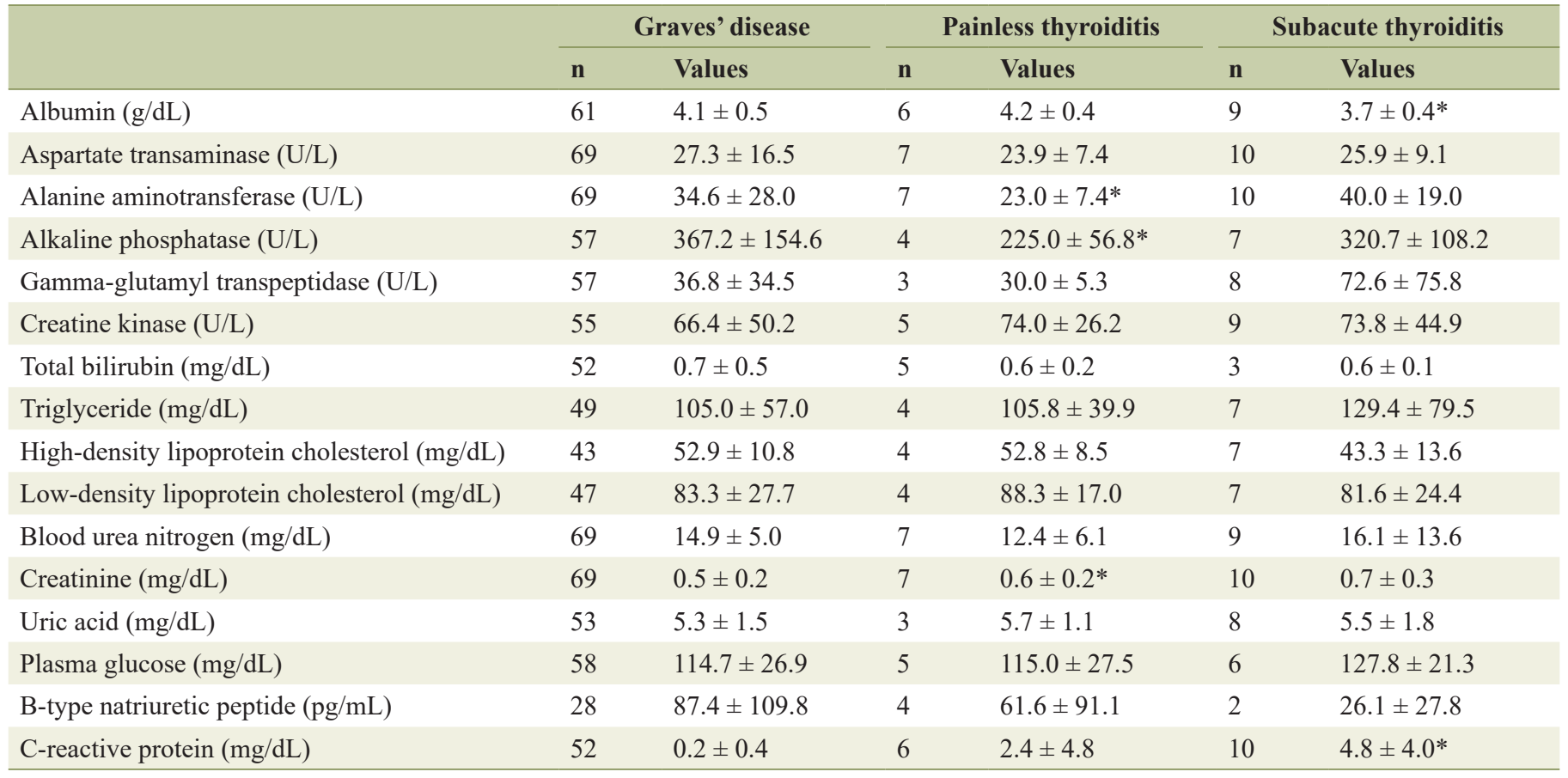

Values indicate mean $\pm \mathrm{SD}$. ${ }^{*} \mathrm{P}<0.05$ versus patients with Graves' disease.

shows that the FT3/FT4 ratio is helpful to exclude painless thyroiditis, which was also observed in our study, supporting a significance of the FT3/FT4 ratio for differentiation between Graves' disease and painless thyroiditis. Hyperthyroidism is an excessive level of thyroid hormones caused by increased synthesis of thyroid hormones, excessive release of preformed thyroid hormones, or an endogenous or exogenous extrathyroidal source. The most common causes of an excessive production of thyroid hormones are Graves' disease, toxic multinodular goiter, and toxic adenoma. The most common cause of an excessive passive release of thyroid hormones is painless thyroiditis [22]. Higher value of the FT3/FT4 ratio may indicate an excessive production of thyroid hormones, and lower value of the FT3/FT4 ratio may indicate excessive release of preformed thyroid hormones.

In our study, WBC count was significantly higher in subacute thyroiditis than in Graves' disease. The percentage of neutrophil among WBC was significantly higher in subacute thyroiditis than in Graves' disease, and the percentages of lymphocyte and eosinophil in subacute thyroiditis were significantly lower than those in Graves' disease. The guideline for diagnosis of subacute thyroiditis presented by the Japan Thyroid Association shows that patients often have preceding episodes of upper respiratory inflammation and high fever. Further, a significant association between subacute thyroiditis and infection has been reported [15-21]. Steroid levels increase immediately after stress such as infection in response to the stimulation of corticotropin-releasing hormone secretion by various cytokines [23]. Steroid causes neutrophilic leukocytosis together with eosinopenia and lymphocytopenia [24]. Hematological changes observed in subacute thyroiditis may be induced by excess steroid release due to increased inflammatory cytokines by infection. High fever is commonly observed in patients with thyroid storm. Remarkably high levels of FT3 $(24.1 \pm 6.0 \mathrm{pg} / \mathrm{mL})$, FT4 $(7.1 \pm 1.8 \mathrm{~g} / \mathrm{dL})$ and the third-generation TRAb $(>30 \mathrm{IU} / \mathrm{L})$ in thyroid storm can make us distinguish between thyroid storm and subacute thyroiditis (FT3, $8.6 \pm 3.3 \mathrm{pg} / \mathrm{mL}$; FT4, $2.9 \pm 0.9 \mathrm{~g} / \mathrm{dL}$ ) [25]. Interestingly, serum CRP level was higher in subacute thyroiditis $(4.8 \pm 4.0$ $\mathrm{mg} / \mathrm{dL})$ than in thyroid storm $(2.2 \pm 1.1 \mathrm{mg} / \mathrm{dL})$ [25].

Serum ALP level in Graves' disease was significantly higher than in painless thyroiditis. This may reflect an increased bone and mineral metabolism due to hyperthyroidism $[26,27]$. Serum CRP level was significantly higher in subacute thyroiditis than in Graves' disease, which is consistent with that elevation of CRP is included in the guideline of diagnosis for subacute thyroiditis by the Japan Thyroid Association. Further, serum albumin level was significantly lower in subacute thyroiditis than in Graves' disease. Lower serum albumin level may be induced by stronger inflammation in subacute thyroiditis [28].

Present study has some limitations. First, the number of studied subjects was small. Second, since this study was retrospective and based on medical charts, lack of data might influence the results. Third, elevated radioactive iodine uptake to the thyroid gland, decreased radioactive iodine uptake to the thyroid gland, and hypoechoic lesion at a painful portion of the thyroid gland confirmed by ultrasonography were included in the guideline for diagnosis of Graves' disease, painless thyroiditis and subacute thyroiditis, respectively. We did not use such diagnostic imaging modalities in diagnosis of these diseases. A more detailed prospective study including a larger 
number of patients is recommended.

\section{Conclusions}

Present study elucidated characteristic clinical and laboratory findings for patients with Graves' disease, painless thyroiditis and subacute thyroiditis who showed hyperthyroidism.

\section{Acknowledgments}

We thank the staff (Yukie Kawamura, Keiko Nakamura, Harue Aoki and Ayano Sakakibara) of the Division of Research Support, National Center for Global Health and Medicine Kohnodai Hospital.

\section{Financial Disclosure}

Authors have no financial disclosure to report.

\section{Conflict of Interest}

The authors declare that they have no conflict of interest concerning this article.

\section{Informed Consent}

Not applicable.

\section{Author Contributions}

$\mathrm{HY}$ and MH designed the research; $\mathrm{MH}$ and $\mathrm{HK}$ collected data; HY analyzed data, and HY wrote the paper. All authors read and approved the final paper.

\section{References}

1. De Leo S, Lee SY, Braverman LE. Hyperthyroidism. Lancet. 2016;388(10047):906-918.

2. Garmendia Madariaga A, Santos Palacios S, GuillenGrima F, Galofre JC. The incidence and prevalence of thyroid dysfunction in Europe: a meta-analysis. J Clin Endocrinol Metab. 2014;99(3):923-931.

3. Hollowell JG, Staehling NW, Flanders WD, Hannon WH, Gunter EW, Spencer CA, Braverman LE. Serum TSH, $\mathrm{T}(4)$, and thyroid antibodies in the United States population (1988 to 1994): National Health and Nutrition Examination Survey (NHANES III). J Clin Endocrinol Metab. 2002;87(2):489-499.

4. Laurberg P, Cerqueira C, Ovesen L, Rasmussen LB, Perrild $\mathrm{H}$, Andersen $\mathrm{S}$, Pedersen $\mathrm{IB}$, et al. Iodine intake as a determinant of thyroid disorders in populations. Best
Pract Res Clin Endocrinol Metab. 2010;24(1):13-27.

5. Kasagi K. [Epidemiology of thyroid tumors: effect of environmental iodine intake]. Nihon Rinsho. 2007;65(11):1953-1958.

6. Vierhapper H, Bieglmayer C, Nowotny P, Waldhausl W. Normal serum concentrations of sex hormone bindingglobulin in patients with hyperthyroidism due to subacute thyroiditis. Thyroid. 1998;8(12):1107-1111.

7. Martino E, Buratti L, Bartalena L, Mariotti S, Cupini C, Aghini-Lombardi F, Pinchera A. High prevalence of subacute thyroiditis during summer season in Italy. J Endocrinol Invest. 1987;10(3):321-323.

8. Fatourechi V, Aniszewski JP, Fatourechi GZ, Atkinson EJ, Jacobsen SJ. Clinical features and outcome of subacute thyroiditis in an incidence cohort: Olmsted County, Minnesota, study. J Clin Endocrinol Metab. 2003;88(5):21002105.

9. Nordyke RA, Gilbert FI, Jr., Lew C. Painful subacute thyroiditis in Hawaii. West J Med. 1991;155(1):61-63.

10. Rao NL, Shetty S, Upadhyaya K, R MP, Lobo EC, Kedilaya HP, Prasad G. Salivary C-Reactive Protein in Hashimoto's Thyroiditis and Subacute Thyroiditis. Int J Inflam. 2010;2010:514659.

11. Alfadda AA, Sallam RM, Elawad GE, Aldhukair H, Alyahya MM. Subacute thyroiditis: clinical presentation and long term outcome. Int J Endocrinol. 2014;2014:794943.

12. Saito S, Sakurada T, Yamamoto M, Yamaguchi T, Yoshida K. Subacute thyroiditis: observations on 98 cases for the last 14 years. Tohoku J Exp Med. 1974;113(2):141-147.

13. Ishihara $\mathrm{T}$, Mori $\mathrm{T}$, Waseda $\mathrm{N}$, Ikekubo K, Akamizu T, Imura H. Histological, clinical and laboratory findings of acute exacerbation of Hashimoto's thyroiditis - comparison with those of subacute granulomatous thyroiditis. Endocrinol Jpn. 1987;34(6):831-841.

14. Nishihara E, Ohye H, Amino N, Takata K, Arishima T, Kudo T, Ito M, et al. Clinical characteristics of 852 patients with subacute thyroiditis before treatment. Intern Med. 2008;47(8):725-729.

15. Mathew J, Jr. Clostridium difficile colitis in the setting of subacute thyroiditis: the chicken or the egg. BMJ Case Rep. 2018;11(1):e226711.

16. Collazos J, Gener B, de Miguel J. Bacterial sinusitis associated with subacute, granulomatous thyroiditis. J Infect. 1997;34(2):158-159.

17. Rathod SB, Tankhiwale SR, Pazare AR. Subacute thyroiditis as consequence of chronic bacterial sinusitis. Lancet. 1991;338(8761):250-251.

18. Schofield PM, Keal EE. Subacute thyroiditis associated with Chlamydia psittaci infection. Postgrad Med J. 1986;62(723):33-34.

19. Mo Z, Dong Y, Chen X, Yao H, Zhang B. Acute transverse myelitis and subacute thyroiditis associated with dengue viral infection: A case report and literature review. Exp Ther Med. 2016;12(4):2331-2335.

20. Martinez-Artola Y, Poncino D, Garcia ML, Munne MS, Gonzalez J, Garcia DS. Acute hepatitis E virus infection and association with a subacute thyroiditis. Ann Hepatol. 2015;14(1):141-142. 
21. Cunha BA, Berbari N. Subacute thyroiditis (de Quervain's) due to influenza A: presenting as fever of unknown origin (FUO). Heart Lung. 2013;42(1):77-78.

22. Kravets I. Hyperthyroidism: diagnosis and treatment. Am Fam Physician. 2016;93(5):363-370.

23. Riad M, Mogos M, Thangathurai D, Lumb PD. Steroids. Curr Opin Crit Care. 2002;8(4):281-284.

24. Fauci AS, Dale DC, Balow JE. Glucocorticosteroid therapy: mechanisms of action and clinical considerations. Ann Intern Med. 1976;84(3):304-315.

25. Yanai H, Hakoshima M, Katsuyama H. Clinical, biochemical, hematological, endocrinological and immunological differences between graves' disease patients with and without thyroid storm. J Clin Med Res. 2019:11(6):452457.

26. Al-Nuaim A, El-Desouki M, Sulimani R, Mohammadiah M. Bone mineral density and metabolic indices in hyperthyroidism. Ann Saudi Med. 1991;11(5):530-533.

27. Cooper DS, Kaplan MM, Ridgway EC, Maloof F, Daniels GH. Alkaline phosphatase isoenzyme patterns in hyperthyroidism. Ann Intern Med. 1979;90(2):164-168.

28. Kaysen GA. Biochemistry and biomarkers of inflamed patients: why look, what to assess. Clin J Am Soc Nephrol. 2009;4(Suppl 1):S56-S63. 